\title{
Stochastic Optimization of Oil Well Placement and Their Type and Trajectory for Maximizing the Net Present Value of Entire Project
}

\author{
Dulian Zeqiraj \\ Faculty of Geology and Mining, Department of Energy Resources, Tirana, Albania
}

\begin{abstract}
The optimal determination of the number of injection and production wells as well as their trajectories in an oil field depends on a series of factors which in most cases are of a stochastic nature. Among the uncertain factors we can mention the geological complexity, combinations of petrophysical, economic parameters, flow regimes, oil type, reservoir rock, sandstone or carbonate. The main reason is to develop optimal algorithms that provide the solution to the problem in a relatively short time or with a relatively small number of iterations. Exactly the latter, time and number of iterations has been a major handicap in the past where it took days to find a solution to the problem. So, the problem is that we should not give greater priority to the speed of computers than the way algorithms are developed in a smart way. The advantages in this regard have been quite good, suffice it to mention the genetic algorithm, particle swarm. So, it is imperative to develop intelligent algorithms that through reservoir simulations give us in a relatively short time, say some hours, the optimal solution to the optimization problem: the location of existing injection and production wells and the location of the trajectory of the candidate wells that will be drilled for both injection and exploitation to achieve the final goal which is the maximization of the Net Present Value. The problem we will pose for solution is of a dual stochastic nature: both in terms of geological uncertainty and economic uncertainty.
\end{abstract}

Keywords: injection and production well trajectory; uncertain geological and economical parameters; reservoir simulation; stochastic optimization. 


\section{Introduction}

The determination of the number of new wells to be drilled in addition to the existing ones (injection and production), their locations, well trajectories is one the main goal in the development plan. Of course, drilling costs for the new wells that need to be drilled also have their own. From the beginning we are emphasizing the fact that our problem is of a stochastic nature both in terms of drilling wells and economically. In terms of well drilling, we are dealing with a complex combination of disciplines including geology, geophysics, drilling engineering and reservoir engineering that in fact geological or geophysical measurements often or almost always contain errors and inaccuracies. It follows that a large number of geological and geophysical realizations are needed. To mention the problem of stochastics in this case, suffice it to say that the price of oil on the stock exchange is uncertain. Regarding the problem of optimization there are already two main methods used; gradient-based (faster in computation) and the other is non-gradient-based. The first author to deal with the gradient-based method was Zandvliet et al. (2008). He used the method of using 8 pseudo-wells (injections) and the well (production) whose placement has to be optimized was surrounded from the wells in question. Net Present Value (NPV) gradients had to be calculated for the entire makespan in correspondence with the rate of injection of the so-called pseudo-wells. This would help in the calculation and also improve the trajectory of the well to have a more accurate location of the well itself. In addition, the authors in question concluded that constraints of production well had an impact on the location and trajectory of the well. Sarma et al. (2008) introduced an interesting method using direct gradients to determine the location of wells. Wange et al. (2012). According to the algorithm developed by the authors, they placed in every cell 1 injection well and then optimizing NPV. To reduce the number of injections wells the authors set as a constraint the drilling costs combined with the amount of injection rate to be used. Yeten et al (2012) introduced a new methodology using genetic algorithm combined with ANN for unconventional wells by which optimized location and trajectory of the wells was archived. 


\section{Methodology}

\section{Drilling cost included in NPV}

According to [1], the objective of this paper is the maximization of NPV and this can be realized after we have found the best trajectory for each well. The NPV of the project is given with the following formula (1)

$$
\begin{aligned}
\mathrm{NPV}=\sum_{k=1}^{K}\left[\begin{array}{l}
\sum_{i=1}^{N_{i n j}} r_{w, i n j} \cdot\left(u_{w, i}\right)_{k}+\sum_{j=1}^{N_{p r}}\left(r_{w, p r} \cdot\left|y_{w, j}\right|_{k}+r_{o, p r} \cdot\left|y_{o, j}\right|_{k}\right) \\
(1+b)^{\frac{t_{k}}{\tau}}
\end{array}\right. \\
\left.\quad \times \Delta t_{k}\right]^{N_{i n j}+N_{p r}} C_{i} \cdot L_{i} b b
\end{aligned}
$$

here,

$b$ - Discount (1/year) Rate,

$C_{i}-$ Cost of drilling per 1 meter, $(\$ / m)$

$k$ - Time step of simulation, -

$L_{i}$ - Trajectory length inside each of gridblocks of the reservoir for every well, $\mathrm{m}$

$N_{\text {inj }}-$ Well's number of injectors, -

$N_{p r}-$ Well's number of producers, -

$r_{o, p r}$ - Oil produced price per volume - unit, $\$ / \mathrm{m}^{3}$

$r_{w, i n j}$ - Water injected cost per volume - unit, $\$ / \mathrm{m}^{3}$

$r_{w, p r}-$ Water produced cost per volume - unit, $\$ / \mathrm{m}^{3}$

$\Delta t_{k}$ - Interval time of each step - time k, day

$\tau$ - Time interval of reference( taken for one year)

$u_{w, i}$ - Rate of water injection for every well $\mathrm{i}, \mathrm{m}^{3} /$ day

$y_{o, j}$ - Rate of oil production for every well j, $\mathrm{m}^{3} /$ day

$y_{w, j}-$ Rate of water production for every well $\mathrm{j}, \mathrm{m}^{3} /$ day

Once the parameterization is complete, a new initial well trajectory is obtained. Here we introduce the concept of dummy wells figure (2). Artificial dummy wells are introduced to improve the direction of the trajectory based on changes in NPV per unit injection. From the block diagram above we note that the gradients can be calculated by performing adjoint backward simulation and forward reservoir simulations. The larger the gradient values the better the trajectory of the well. Now the question is whether these trajectories can be drilled, which is ensured by constraints of drillability. To determine trajectory of the wells in the sense of optimality, i.e., consequently its location, as a procedure, the production well must be surrounded by an imaginary pseudo-side track. In this way based on the gradients we are able to get to the optimal solution for the whole trajectory. Since dummy wells produce or inject with low debits, then they do not affect or affect the overall flow in the reservoir very little. 


\section{SABASECONF}

02-04 JULY, 2021

Oxford, United Kingdom

Basically, the dummy wells help to improve the direction, which in turn as the trajectory of the well changes to improve the objective function

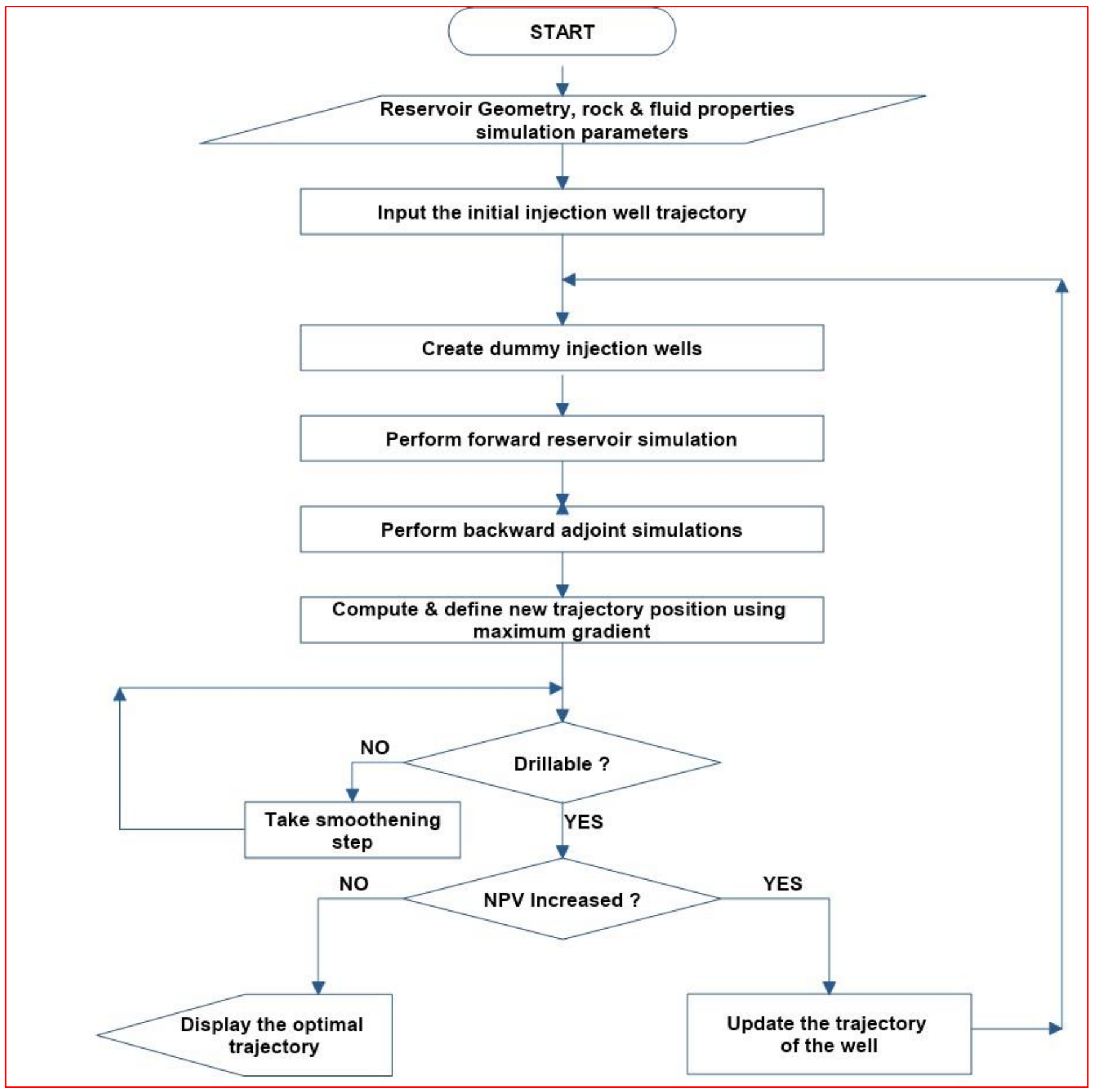

Figure 1. An Injection Overview of the trajectory of the well for the process of optimization (A. A.

Shamsuddeen, 2017, \& D. Zeqiraj 2021) 


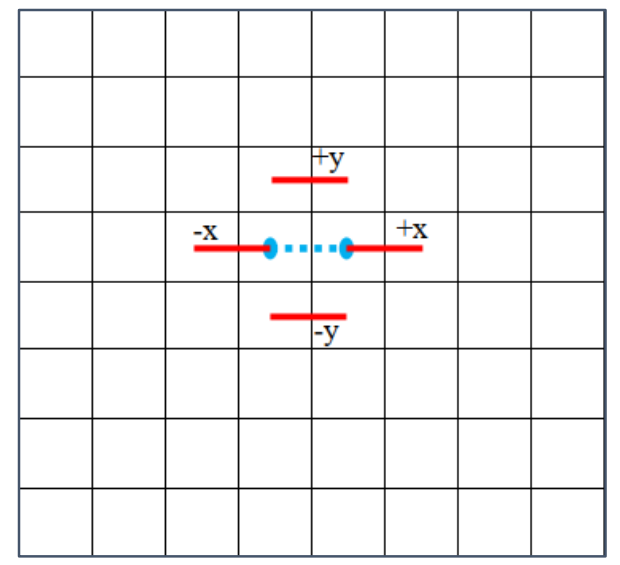

Figure 2. Concept of dummy wells, where a horizontal well (blue color) is surrounded by 4 dummy wells (D. Zeqiraj, MRST)

\section{Improving Direction and Revised Position}

The following figure 3 shows the block diagram of the algorithm where by accepting an initial trajectory, this algorithm delineates a new trajectory, which in turn provides us with an increase in NPV. In other words, by improving the direction of the well, a higher value of the objective function is achieved.

For each segment of the well we generate the dummy wells and, in this way:

The formula is:

$$
g=\sum_{k=1}^{K} \frac{\partial N P V}{\partial u_{d, k}}
$$

Here $g$ is the summed gradient, $u_{d, k}$ is the flowrate $u$ at time-step $k$ in dummy well $d$ and $K$ is total of time-step for all the simulation. The selected dummy wells (with the highest gradients) are then used for improving the location of well trajectory based to a weighing factor $\beta$.

$$
x_{i}^{j+1}=\beta p_{i}^{j}+(1-\beta) x_{i}^{j}
$$

In the formula above $\mathrm{x}$ yields the vector (position) of the $i^{\text {th }}$ trajectory point, $j$ is the iteration and $\beta$ is the step size corresponding to the improved direction. 
Oxford, United Kingdom

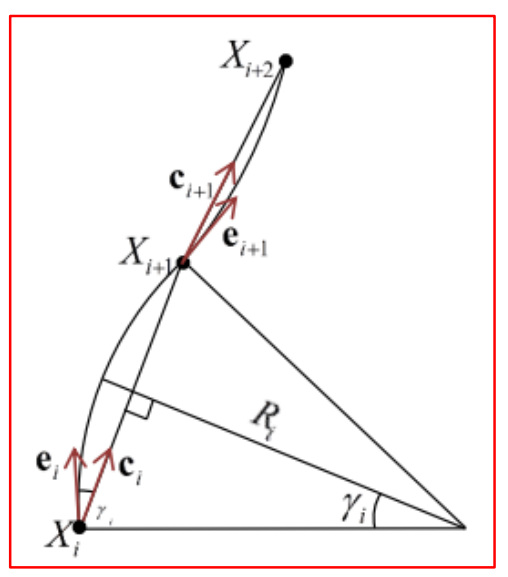

The figure on the left display's trajectory as a sequence of each of curvature with different radii. In the figure we have two consecutive curvature-turns with different radii. In short, if we are going to refer to figure 1 , the question of whether the well is drillable or not and assuming that the curvature 1 with large radius is undrillable then in the algorithm and consequently in the programming in MATLAB we pass to the next second curvature of which turns out to be drillable. The procedure is repeated as here and even to situations similar to the figure occur.

Figure 3. The sequence of the curvature 
$3^{\text {rd }}$ International Conference on Advanced Research in Applied SCIENCE \& ENGINEERING

02-04 JULY, 2021

Oxford, United Kingdom

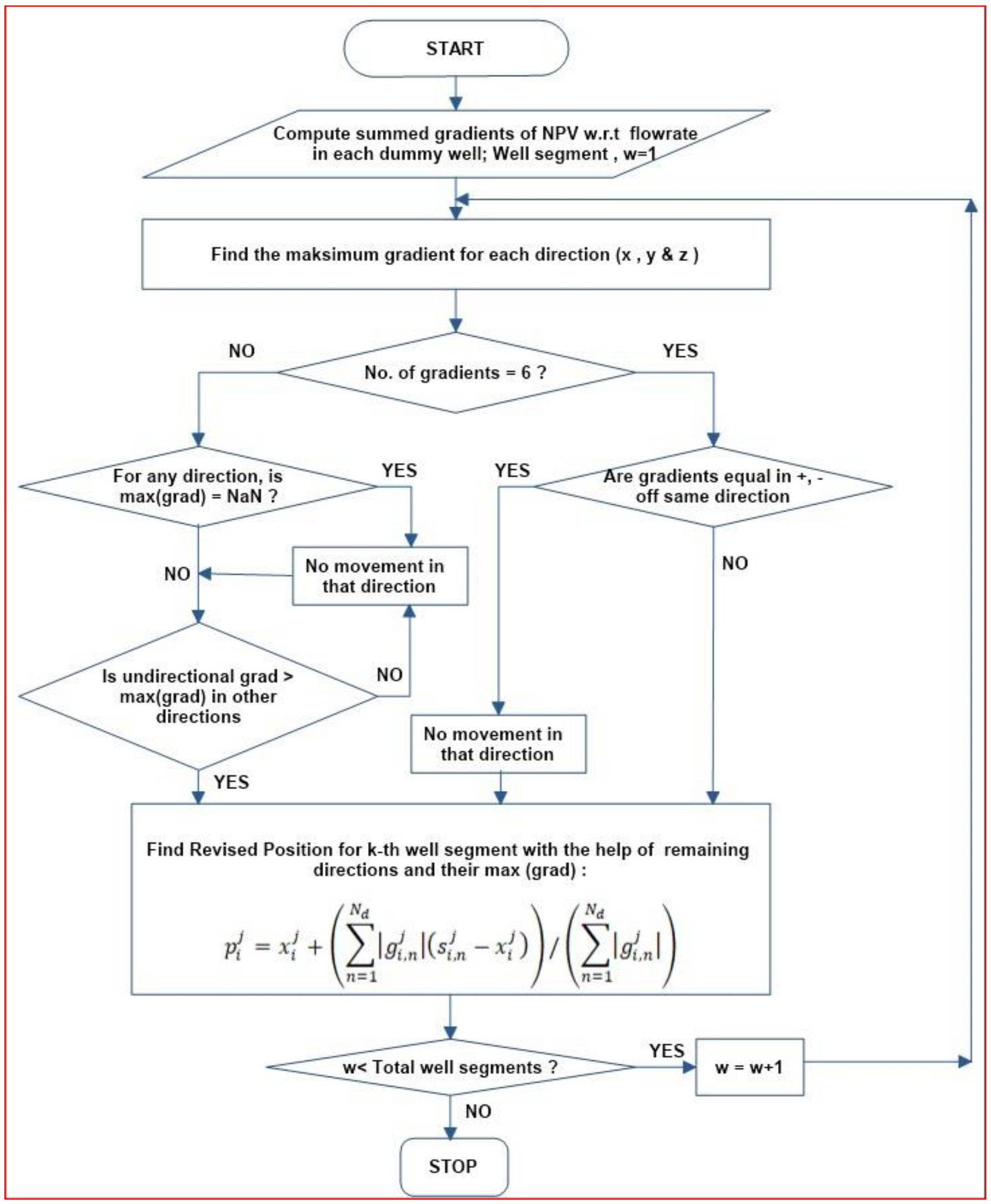

Figure 4: An computation overview of position of all the segments well (A. A. Shamsuddeen, 2017 \& D. Zeqiraj 2021) 
$3^{\text {rd }}$ International Conference on Advanced Research in Applied SCIENCE \& ENGINEERING

\section{S(EASECONF}

02-04 JULY, 2021

Oxford, United Kingdom

\section{Implementation \&Results}

We have implemented the algorithms of figures 1 and 3 of the author A. A. Shamsuddeen (2017) with some small appendices of the author of this paper, D. Zeqiraj (2021) in MATLAB. By using the opensource MRST the author of this paper has made the appropriate changes, respectively. The model of flow is black-oil. In the following figure 4 we have (from MRST-MATLAB and D. Zeqiraj) a semitransparent representation of 2 drilled wells, in a sandstone reservoir containing black-oil. The well in the left of the figure 4 is a multilateral production well (comb-like well) and in the right of figure we have "drilled" the segmented injection well (a forked well). Once again, we are reiterating the fact that the drilling of the two wells in figure 4 is made possible by the programming in MATLAB-MRST referring to the algorithms in figures 1 and 3. The injection agent is water and the simulation time, for which the figure is represented is 73-day, 1 hour, 590 seconds. To make the figure more understandable, we "removed" the upper part of the reservoir rock leaving a layer 2 meters high. We did this precisely because during the initial time mentioned above for the simulation (73 days, 1 hour and 590 seconds) in the remaining bottom layer a yellow area can be noticed near the injection well which represents the saturation of the water phase. The reader may ask the logical question of how a 5000x4000x $10 \mathrm{~m}$ reservoir is made available to a development plan with "only" two wells. It so happened that we have planned a 20-year development plan and utilization with relatively high debits.

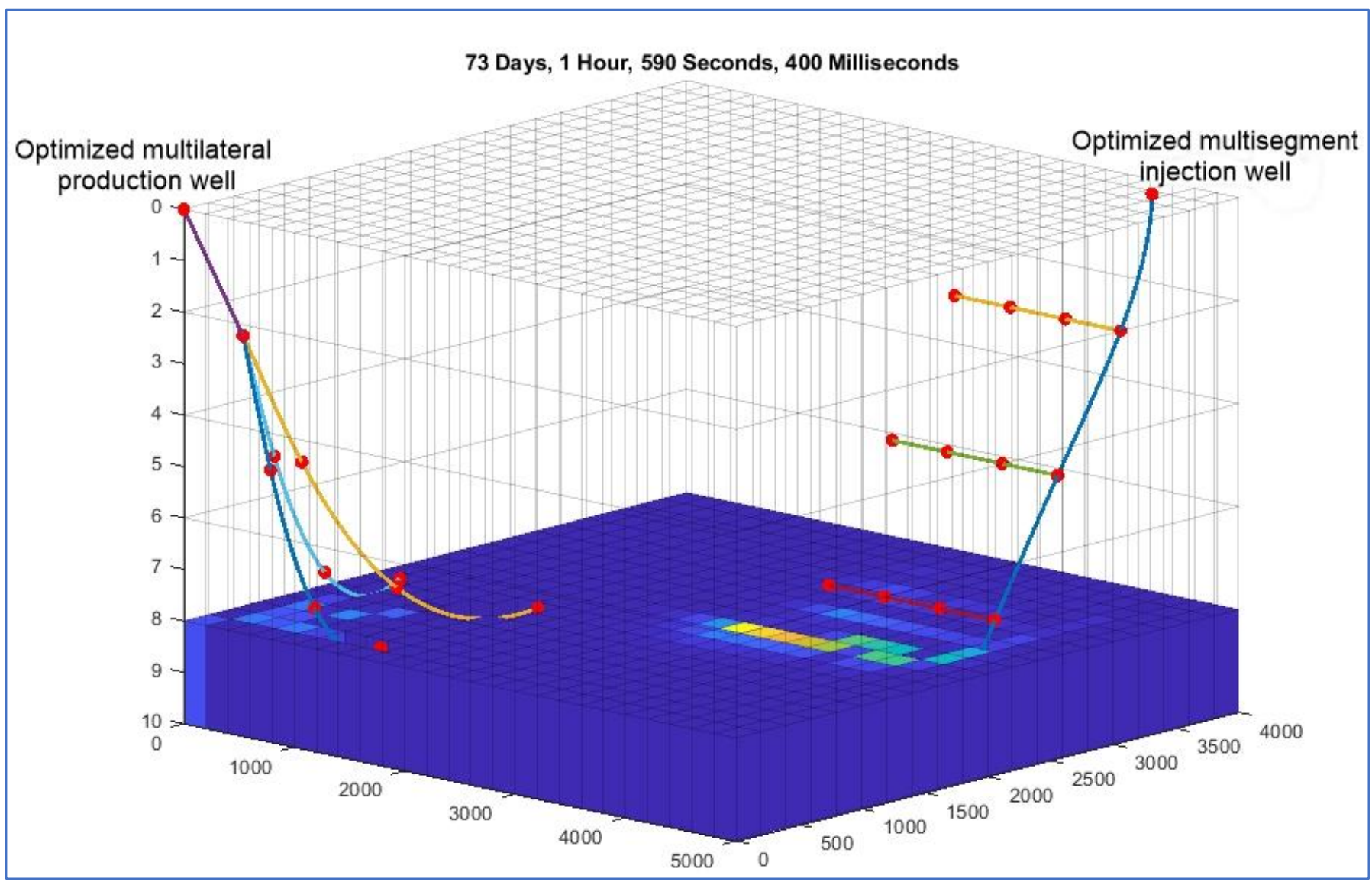

Figure 5. Semi-transparent representation of 2 drilled wells, in a sandstone reservoir containing blackoil (MRST-MATLAB \& D. Zeqiraj). Everything represented here (for the wells), the locations, design, 


\section{S耍ASECONF}

02-04 JULY, 2021

Oxford, United Kingdom

the number of wells, trajectories, configuration are made possible from the objective function, that is maximizing the stochastic NPV for 20 years of exploitation (D. Zeqiraj, MRST)

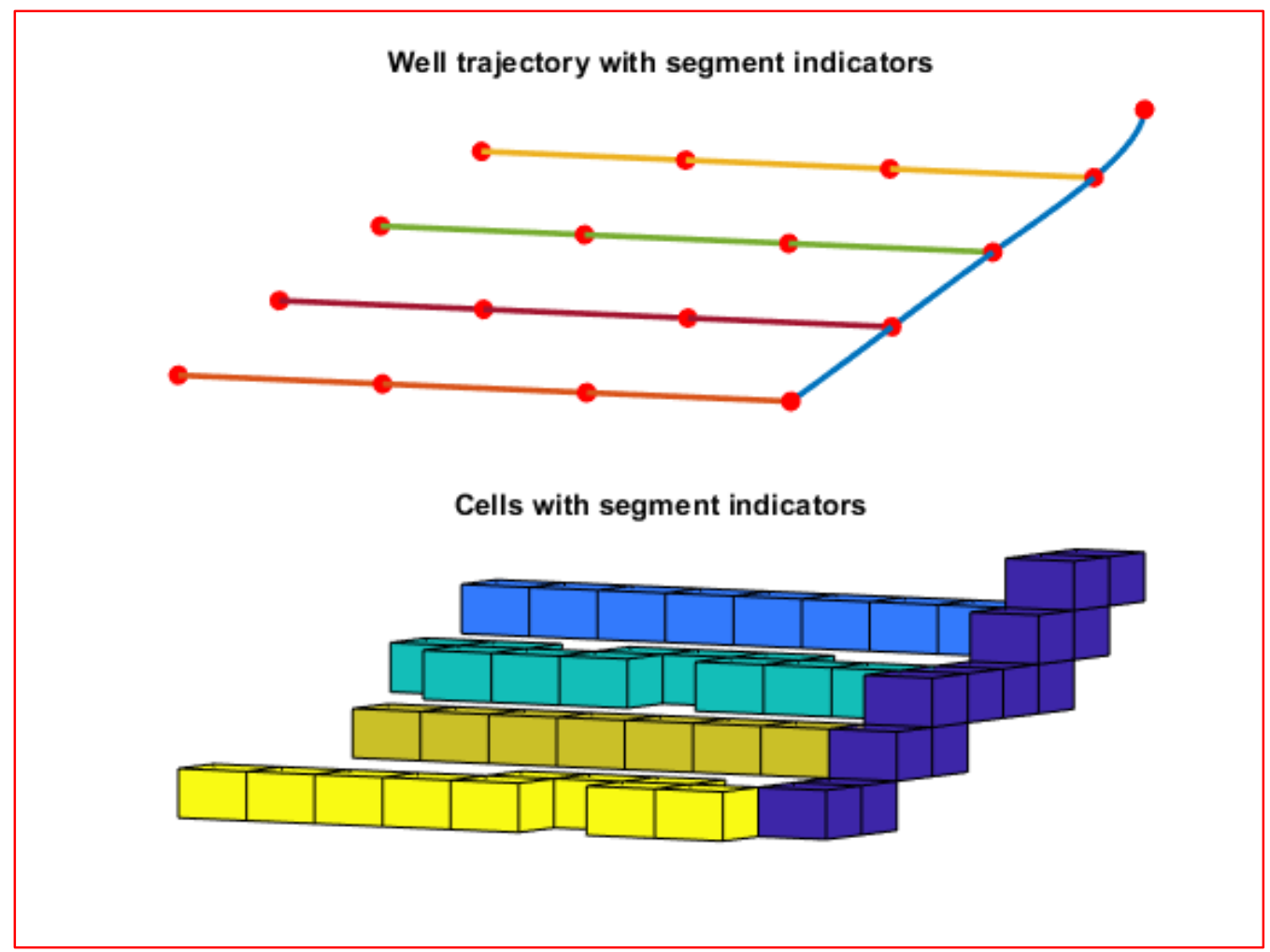

Figure 6. An overview of the injection multilateral well. The result of this design and the coordinates of placement are obtained from optimization and implementation in MATLAB of diagrams in figures 1 and 2 (D. Zeqiraj, MRST) 


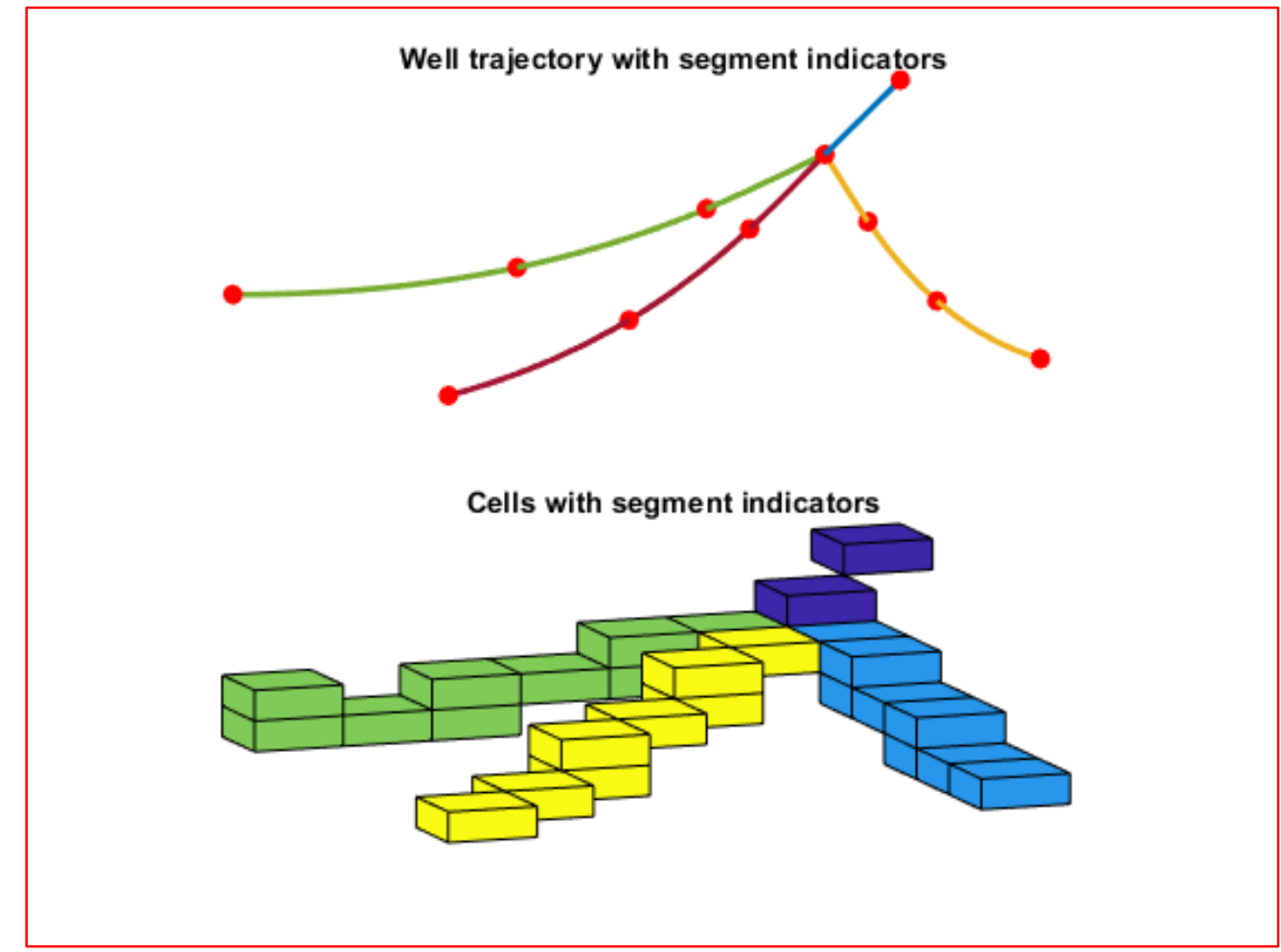

Figure 7. An overview of the production multilateral well. The result of this design and the coordinates of placement are obtained from optimization and implementation in MATLAB of diagrams in figures 1 and 3 (D. Zeqiraj, MRST) 
Oxford, United Kingdom

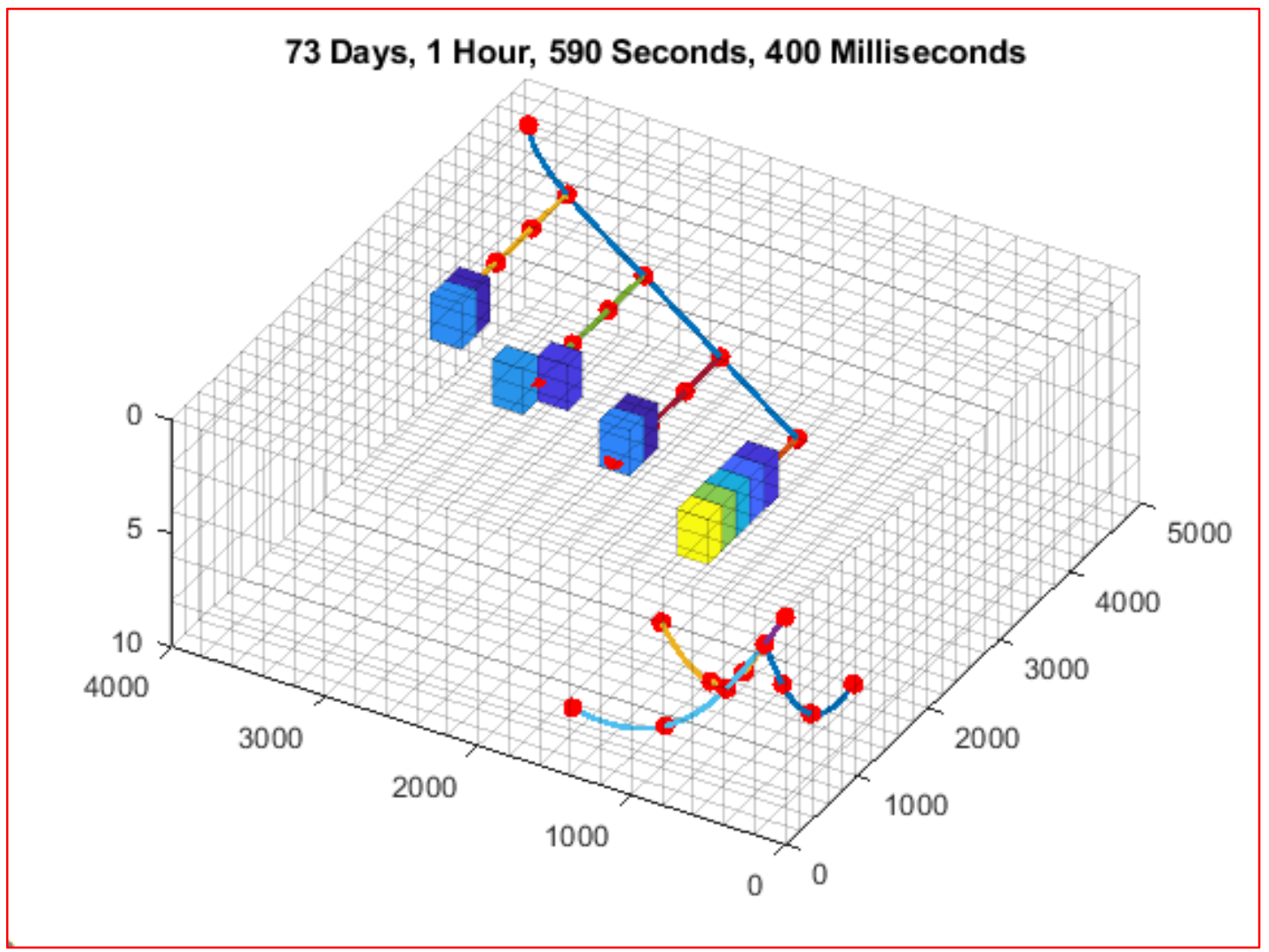

All the figures in this paper are as a result of programing in MATLAB

Type of fluid in reservoir is black-oil and the agent of injection is water.

Injection has just begun only 73 days, 1 hour

Total time of simulation is 20 years

The front of oil has not arrived yet in the production well

Figure 8. Injection well on the upper part, production well near origin. Both the wells are multilateral and this configuration result from optimization procedure. Time of simulation 73 Days-1 Hour-590 sec (D. Zeqiraj, MRST) 


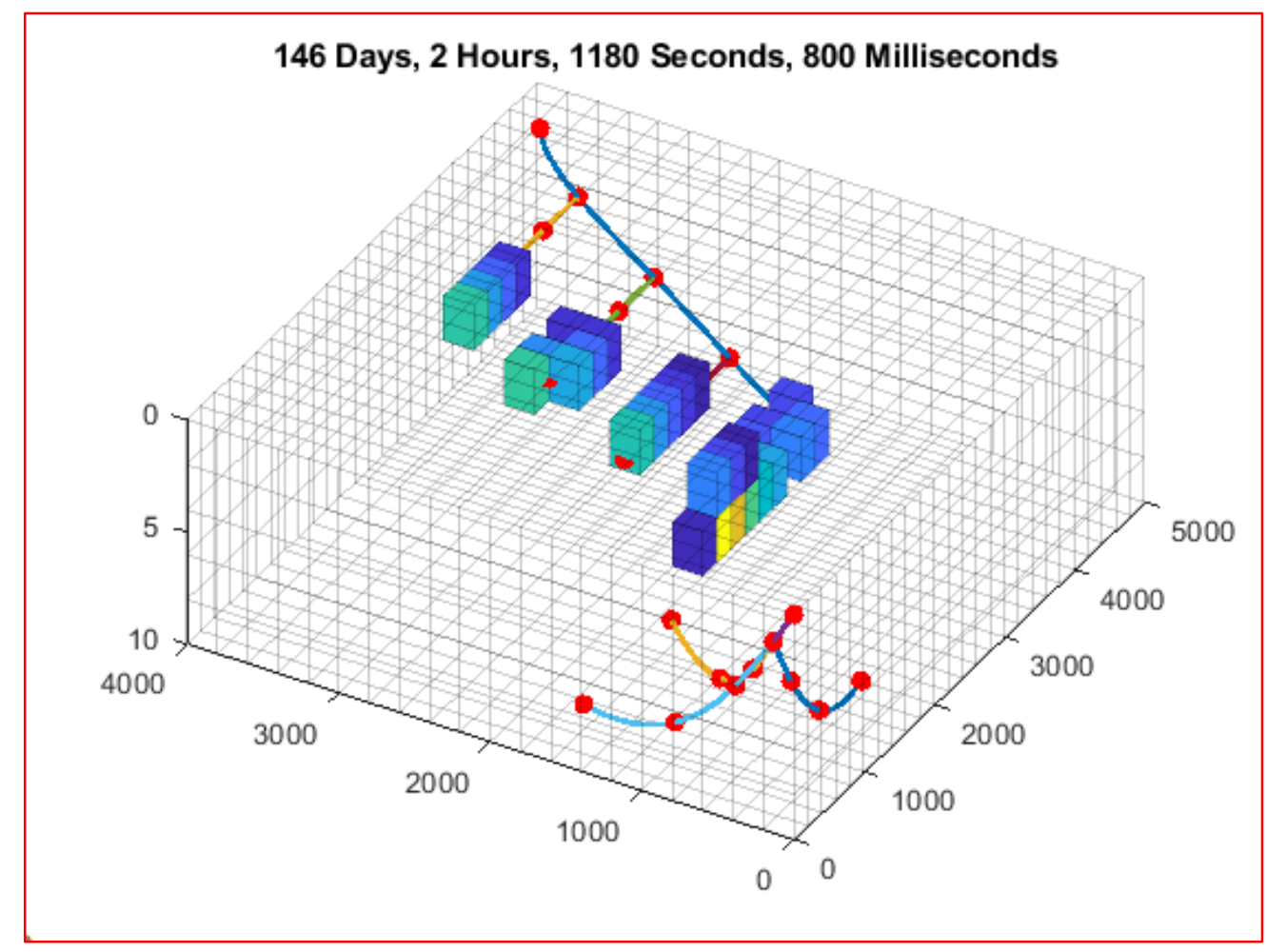

Figure 9. The saturation front of oil after a double period of time (146 days) from the injection well (D. Zeqiraj, MRST) 

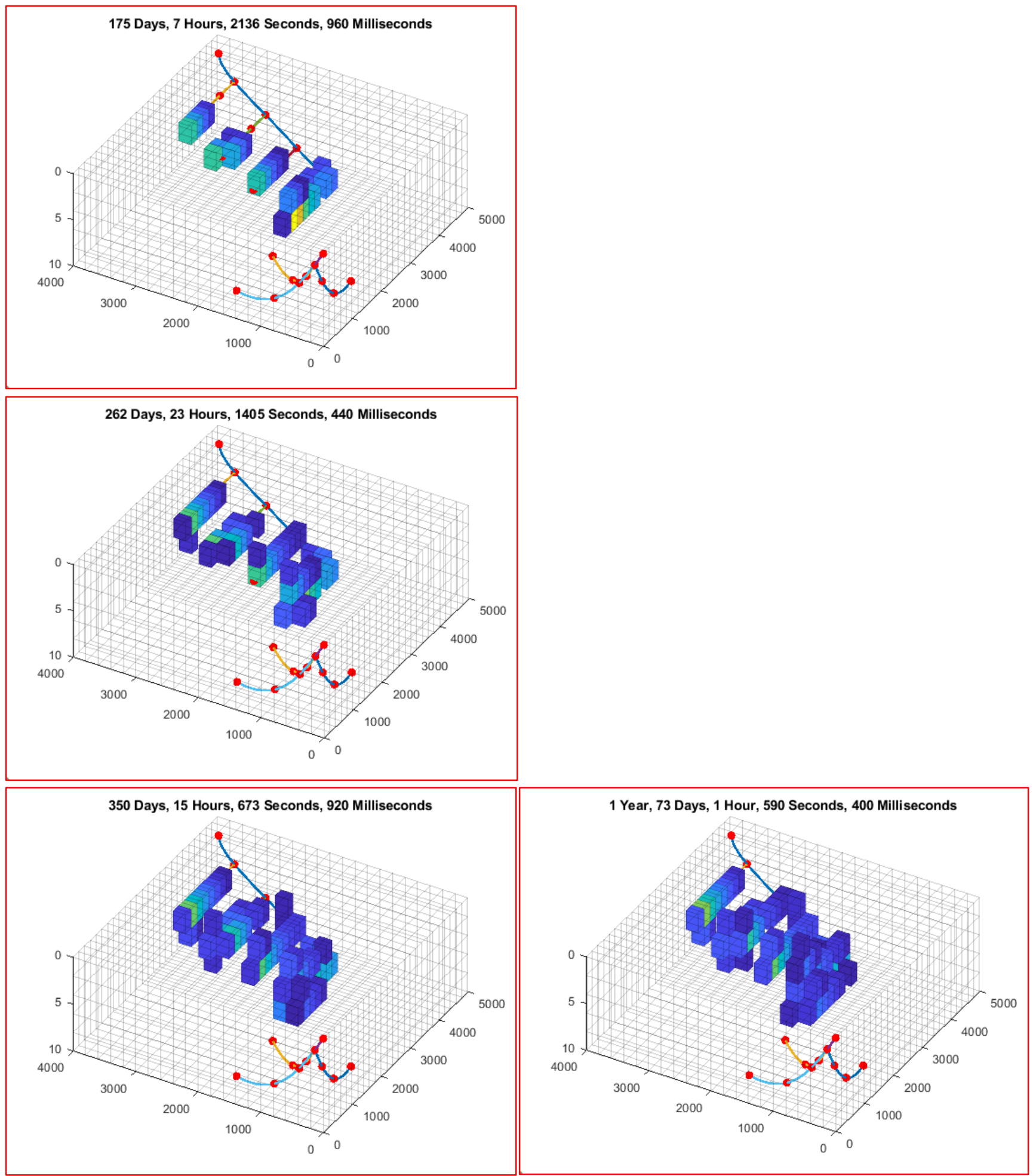

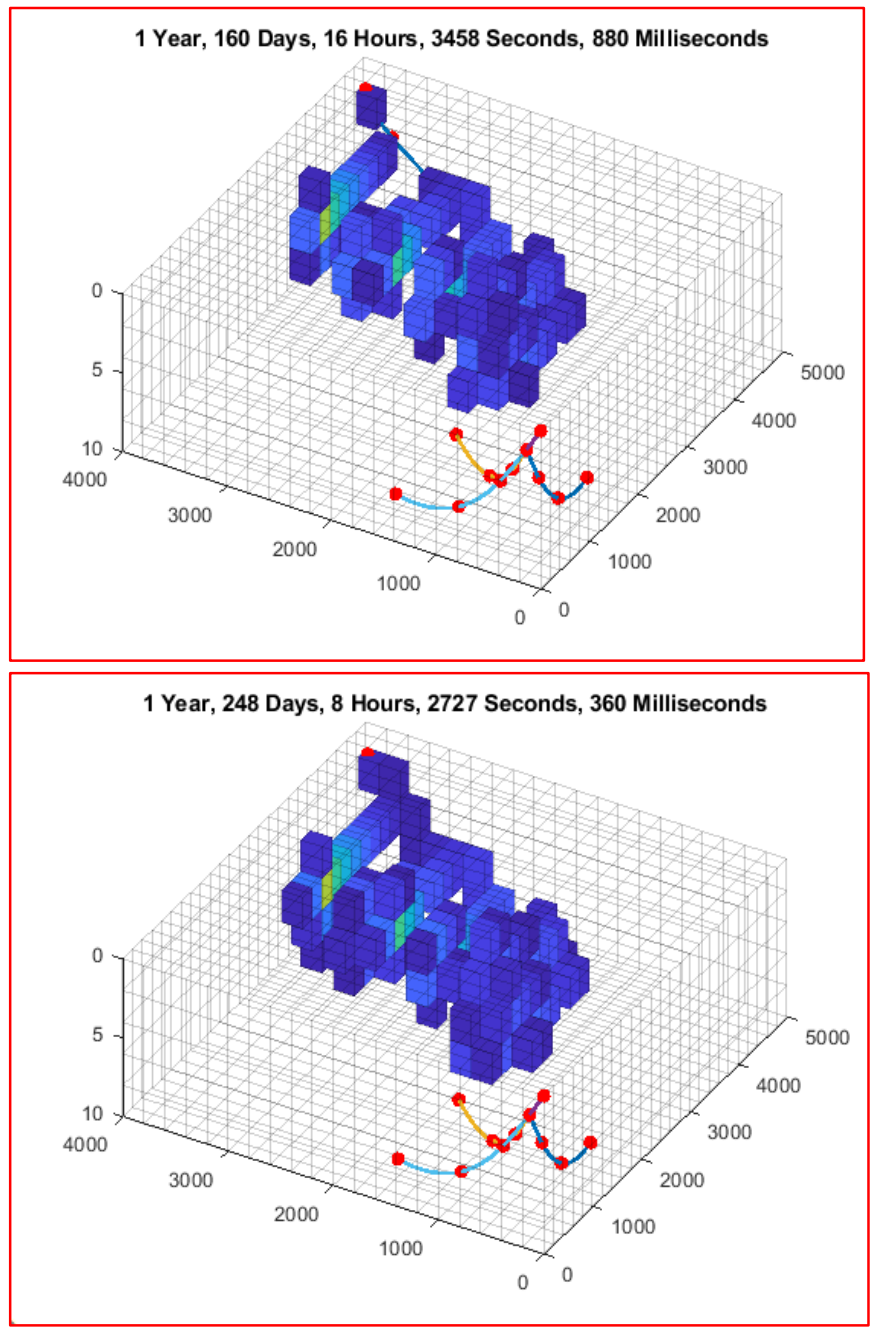

Figure 10. The evolution of the saturation front of fluid for different progressive time, programing in MATLAB (D. Zeqiraj, MRST) 


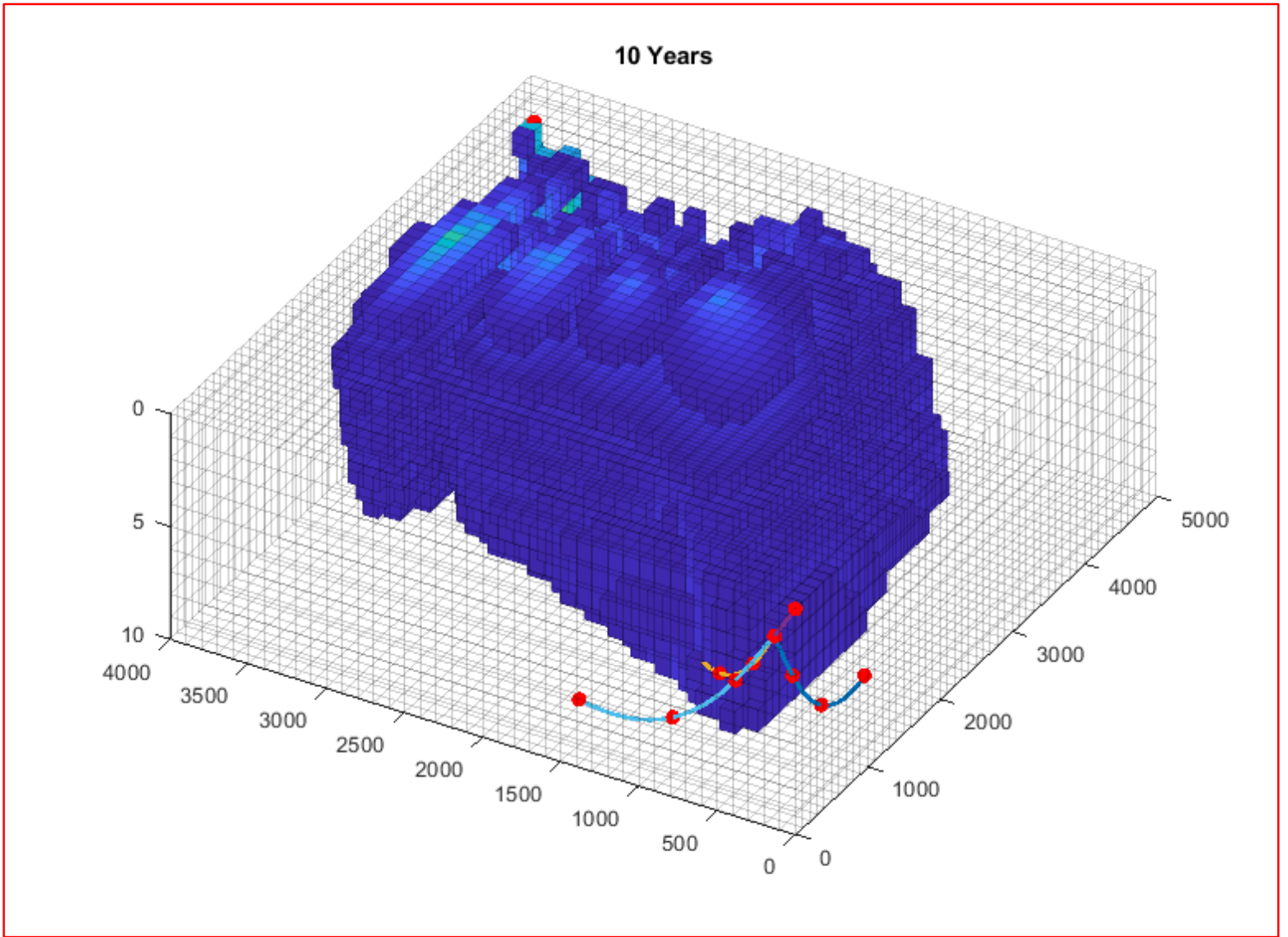

Figure 11. The evolution of the saturation front of fluid for the simulation time $=10$ years (D. Zeqiraj, MRST)

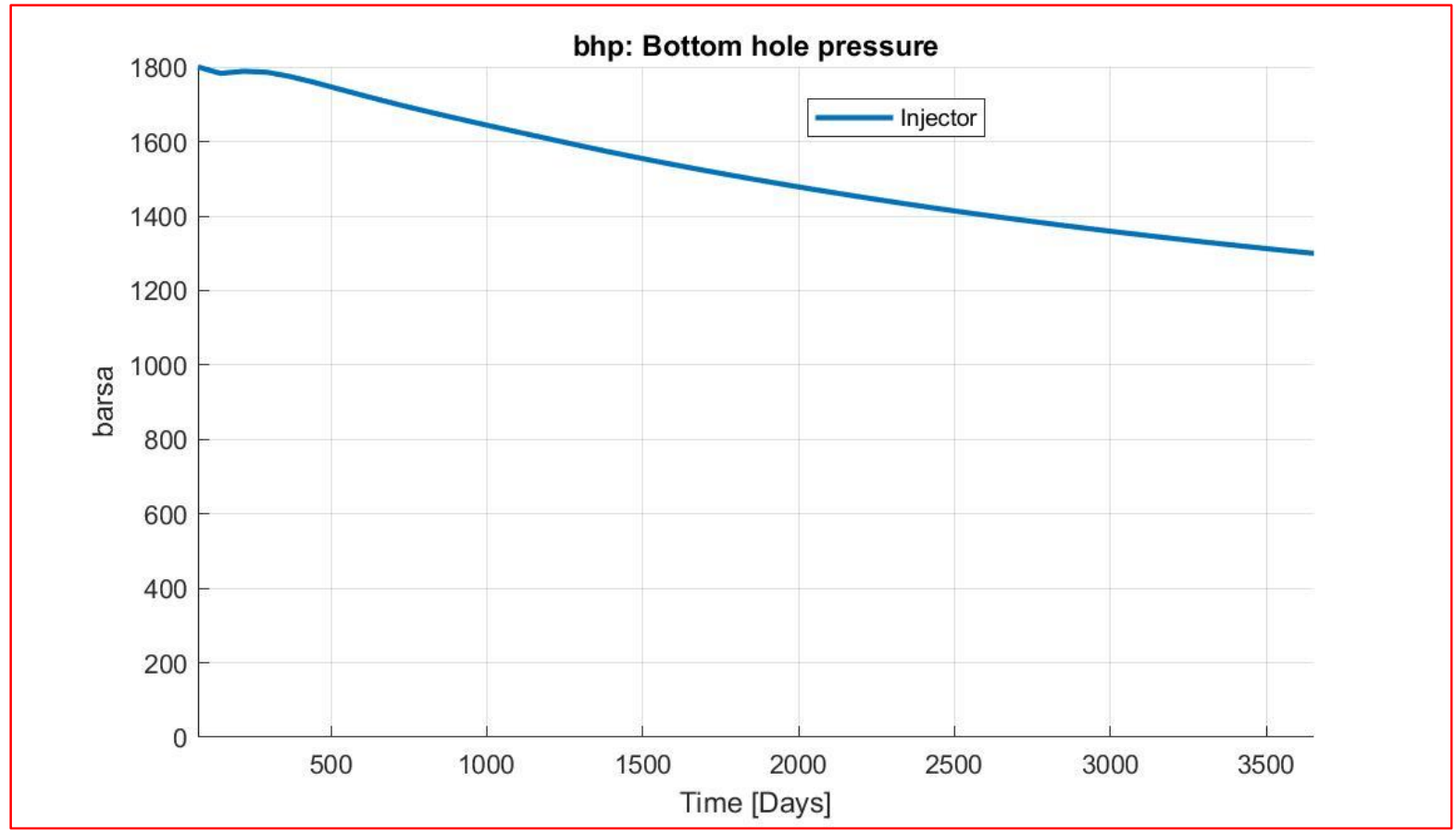

Figure 12. Bottom hole pressure for the injection well for a half lifespan of 10 years (D. Zeqiraj, MRST) 


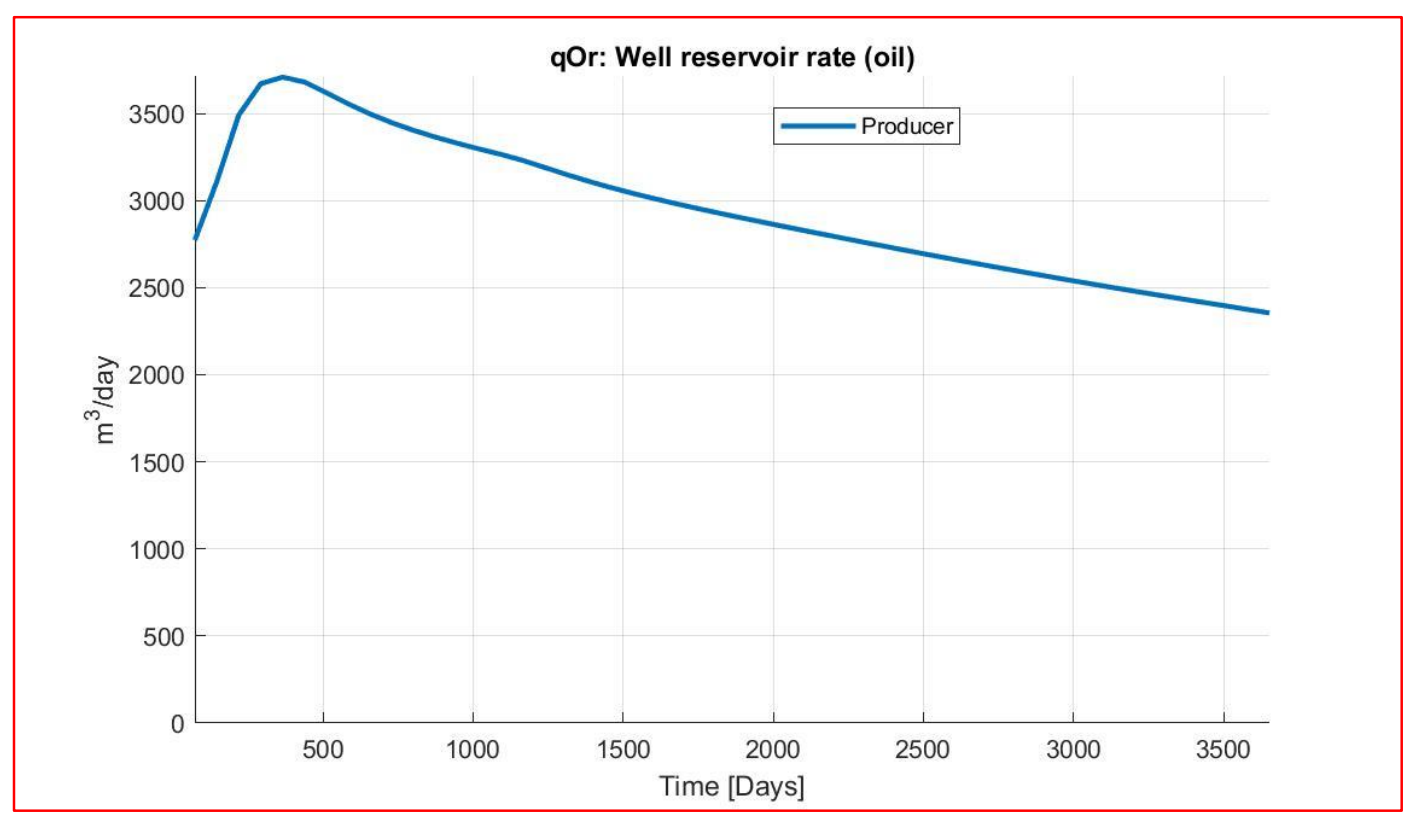

Figure 13. The evolution of the well reservoir rate (oil) for the production well for a half lifespan of 10 years (D. Zeqiraj, MRST)

\section{Discussion}

Our problem in question lacks a significant and necessary element, that of the upscaled model in the conditions we have presented for solving our problem. This happened for technical reasons due to the size of the problem and the optimization procedures. Since the author for the first time in one of his works has decided to include history matching to correct problems that arise (and inevitably) during the upscale of the model in the classical way, the time required for calculations on the computer was prohibited. But the benefits in this case are quite significant making it possible to avoid the errors we make during standard upscale procedures. As an illustration let us refer to the following example which also shows the rapid convergence of the optimization procedure. Smart solutions must finally be found for rapid programming of the problem in question. For the future this will be included with significant effort in our main problem. (Remember that history matching is time-consuming) 


\section{Example}

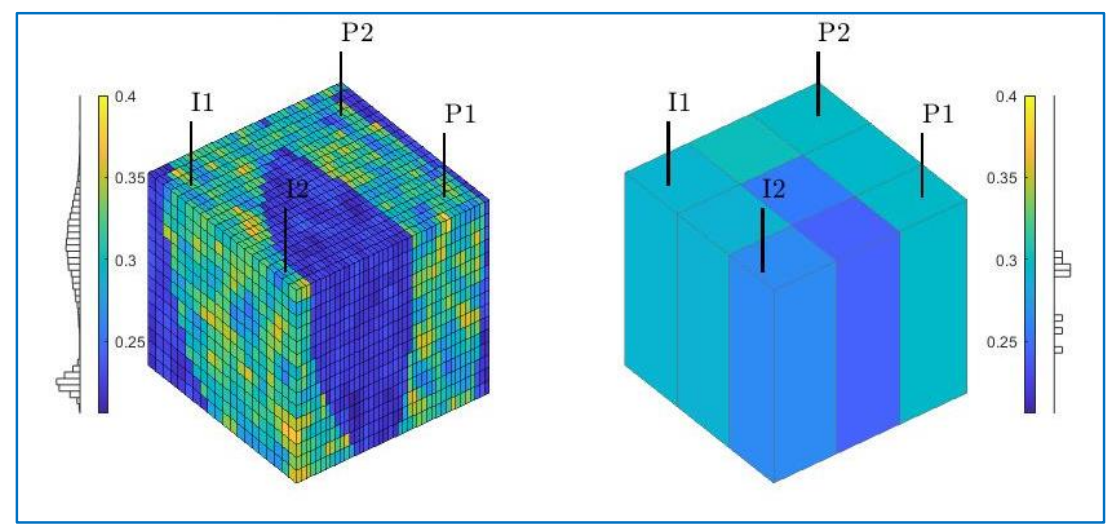

Figure 14. In the left the fine original model, in the right the classic upscaled coarse model with the injections and producer wells (D. Zeqiraj, MRST)

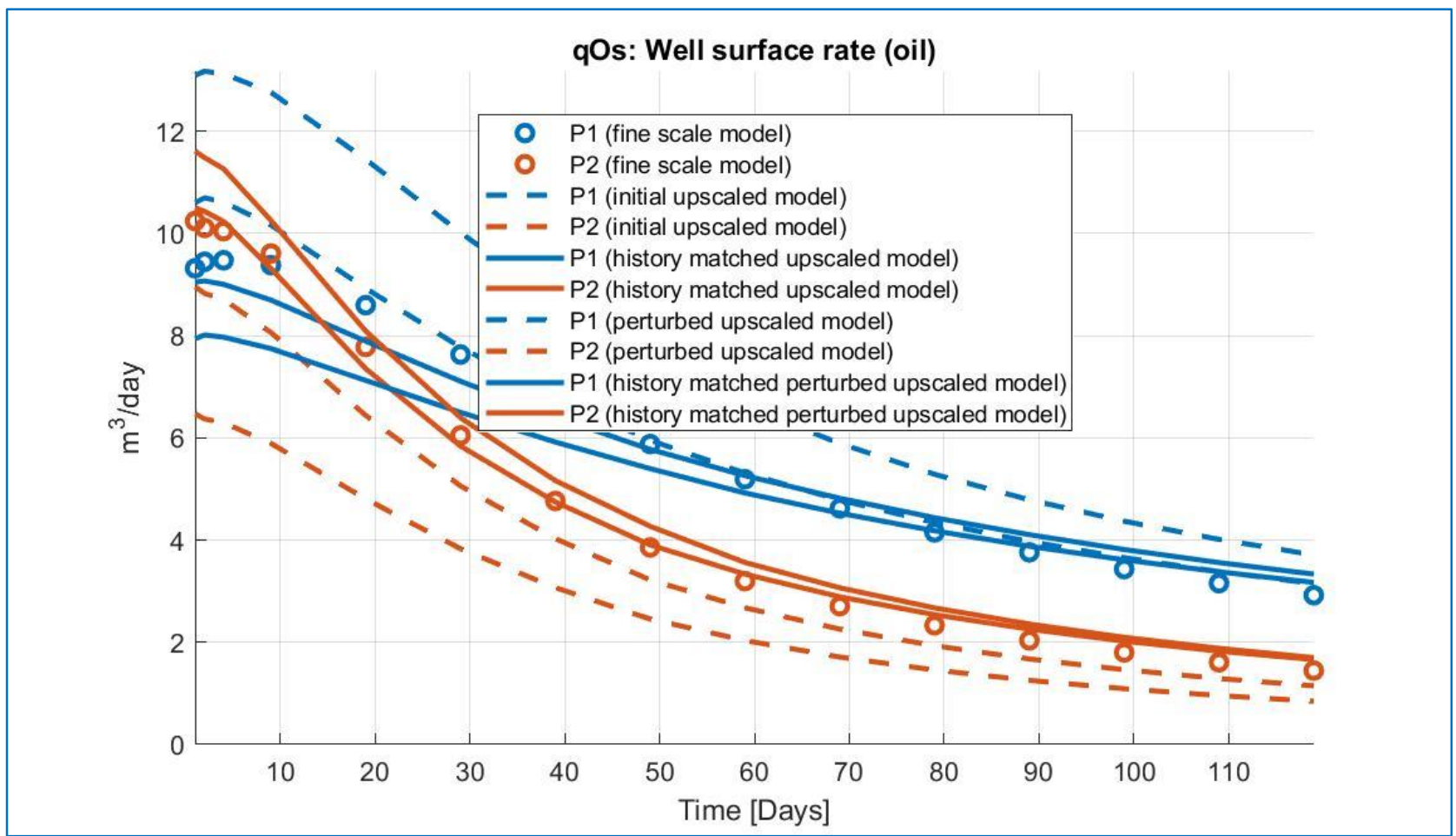

Figure 15. In standard methods of upscale procedure there is a gap between fine scale and coarse scale. From the figure you can see that when including history matching the approximation with the original fine scale model is better than with the classic coarse-upscale model. Lastly observe the well surface rate vs time (D. Zeqiraj, MRST) 


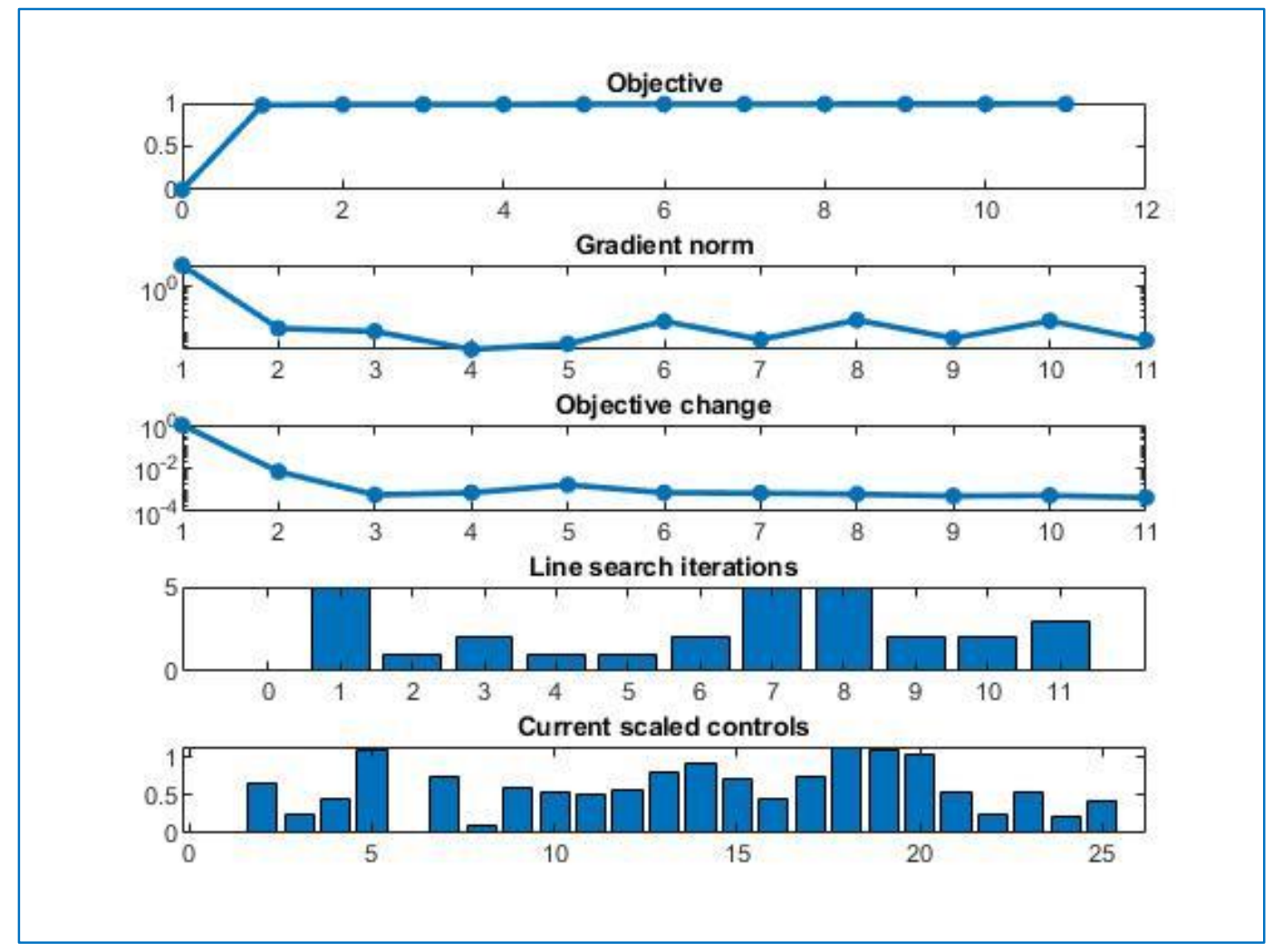

Figure 16. Long time convergence of the history matching inclusion in the programming (D. Zeqiraj, MRST) 


\section{Conclusions}

In this paper the author has made possible to create or rather to reduce the algorithms-block diagrams in figures 1 and 3 by adding some additional elements and bypassing others. Optimizing the placement of injection and production wells is not easy, at least if we refer to the literature. It is more evident that is more difficult when we have to deal with multilateral wells trajectory. The author has managed to avoid inverse problems since they are in most cases ill-conditioned. In the problem of finding the optimal location of wells and their trajectories, the priority in choosing an objective function that does not take into account in terms of whether a well should be deviated or not is good news. To be realistic a lot of work must be performed in formulating as many details and terms as possible in the objective function and constraints. Also, the inclusion of history matching even though it is time-consuming is an indispensable tool to have reliable and real results.

\section{References}

[1] A. A. Shamsuddeen, H. Ismail, Z. Z. Ibrahim, (2017). "Well Trajectory Optimization of Homogeneous and Heterogeneous Reservoirs by the Use of Adjoint Based Optimization Technique"

[2] Aitokhuehi, I. and Durlofsky, L.J. 2005. Optimizing the performance of smart wells in complex reservoirs using continuously updated geological models. J. Pet. Sci. Eng. 48 (3-4): 254-264. http://dx.doi.org/10.1016/ j.petrol.2005.06.004.

[3] Artus, V., Durlofsky, L.J., Onwunalu, J.E., and Aziz, K. 2006. Optimization of nonconventional wells under uncertainty using statistical proxies. Comput. Geosci. 10 (4): 389-404. http://dx.doi.org/10.1007/s10596-006-9031-9.

[4] Bangerth, W., Klie, H., Wheeler, M.F., Stoffa, P.L., and Sen, M.K. 2006. On optimization algorithms for the reservoir oil well placement problem. Comput. Geosci. 10 (3): 303-319. http://dx.doi.org/10.1007/s10596-006-9025-7.

[5] Chen, H. and Schmeiser, B.W. 2001. Stochastic root finding via retro- spective approximation. IIE Trans. 33 (3): 259-275. http://dx.doi. org/10.1080/07408170108936827

[6] Chen, Y., Oliver, D.S., and Zhang, D. 2009. Efficient Ensemble-Based Closed-Loop Production Optimization. SPE J. 14 (4): 634-645. SPE- 112873-PA. http://dx.doi.org/10.2118/112873-PA.

[7] Eberhardt, R.C. and Kennedy, J. 1995. A new optimizer using particle swarm theory. Proc., Sixth International Symposium on Micro Machine and Human Science (MHS '95), Nagoya, Japan, 4-6 October, 39-43. http://dx.doi.org/10.1109/MHS.1995.494215.

[8] Güyagüler, B. and Horne, R.N. 2004. Uncertainty Assessment of Well- Placement Optimization. SPE Res Eval \& Eng 7 (1): 24-32. SPE 87663. http://dx.doi.org/10.2118/87663-PA

[9] Kelley, C.T. 1999. Iterative Methods for Optimization. Philadelphia, Penn- sylvania: Frontiers in Applied Mathematics, SIAM 
[10] Kennedy, J. and Eberhardt, R.C. 1995. Particle swarm optimization. Proc., 1995 IEEE International Conference on Neural Networks, Perth, West- ern Australia, 27 November-1 December, 1942-1947.

[11] Onwunalu, J.E. and Durlofsky, L.J. 2010. Application of a particle swarm optimization algorithm for determining optimum well location and type. Comput. Geosci. 14 (1): 183-198. http://dx.doi.org/10.1007/ s10596-009-9142-1.

[12] Onwunalu, J.E. and Durlofsky, L.J. 2011. A New Well-Pattern-Optimiza- tion Procedure for LargeScale Field Development. SPE J. 16 (3): 594-607. SPE-124364-PA. http://dx.doi.org/10.2118/124364PA.

[13] Özdogan, U. and Horne, R.N. 2006. Optimization of Well Placement Under Time-Dependent Uncertainty. SPE Res Eval \& Eng 9 (2): 135-145. SPE-90091-PA. http://dx.doi.org/10.2118/90091-PA.

[14] D. G. Krige. A statistical approach to some basic mine valuation problems on the witwatersrand. Journal of the Chemical, Metallurgical and Mining Society, 52:119-139, 1951.

[15] H. Langou“et. optimisation sans d'eriv'ees sous contraintes. PhD thesis, Universit'e Nice Sophia Antipolis, June 2011.

[16] C. Li and P. H. Heinemann. A comparative study of three evolutionary algorithms for surface acoustic wave sensor wavelength selection. Sensors and Actuators B: Chemical, 125(1):311 - 320, 2007.

[17] O. Maron and A. W. Moore. The racing algorithm: Model selection for lazy learners.

Artificial Intelligence Review, 11:193-225, 1997. 10.1023/A:1006556606079.

[18] N. S. Mera. Passive gamma tomography reconstruction of layered structures in nuclear waste vaults. Inverse Problems, 23(1):385 - 403, 2007.

[19] Z. Michalewicz. Heuristic methods for evolutionary computation techniques. Journal of Heuristics, 1(2):177-206, 1996.

[20] Z. Michalewicz, D. Dasgupta, R. G. L. Riche, and M. Schoenauer. Evolutionary al- gorithms for constrained engineering problems. Computers \& Industrial Engineering Journal, 30(4):851-870, 1996.

[21] Z. Michalewicz and G. Nazhiyath. Genocop III: a co-evolutionary algorithm for nu- merical optimization problems with nonlinear constraints. In D. B. Fogel, editor, Proceedings of the Second IEEE International Conference on Evolutionary Compu- tation, pages 647-651, Piscataway, NJ, USA, 1995. IEEE Press.

[22] G. Montes, P. Bartolome, and A. L. Udias. The use of genetic algorithms in well placement optimization. In SPE Latin American and Caribbean Petroleum Engi- neering Conference, number SPE 69439, March 2001.

[23] A. Morales, H. Nasrabadi, and D. Zhu. A modified genetic algorithm for horizontal well placement optimization in gas condensate reservoirs. In SPE annual technical conference and exhibition, number SPE 135182, September 2010. 
[24] L. Nakajima and D. J. Schiozer. Horizontal well placement optimization using quality map definition. In Canadian International Petroleum Conference, number 2003-053. Petroleum Society of Canada, June 2003.

[25] J. A. Nelder and R. Mead. A simplex-method for function minimization. Computer journal, 7:308$313,1965$. 